\title{
A Modelica VSC-HVDC Average Value Model Implementation and its Software-to-Software Validation using an EMT Power System Domain Specific Simulator
}

\author{
Mohammed Ahsan Adib Murad $^{1} \quad$ Luigi Vanfretti $^{2}$ \\ ${ }^{1}$ School of Electrical Engineering, University College Dublin, Ireland, mohammed.murad@ucdconnect . ie \\ ${ }^{2}$ School of Electrical Engineering, KTH Royal Institute of Technology, Sweden, luigiv@kth. se
}

\begin{abstract}
This paper reports the implementation of a three-phase VSC-HVDC model using the Modelica language. The model is suitable for power system simulation where the power electronic circuitry can be represented using equivalent voltage and current sources to model the high frequency switching process. Differently from the authors previous work, this model is built using as much components as possible from the MSL (Modelica Standard Library) to represent the three-phase electrical circuit, while implementing the de facto control system models used within typical power system simulation tools. To show the applicability of Modelica for modeling a VSC-HVDC, a software-to-software validation is performed using the EMTP-RV power system simulator.
\end{abstract}

Keywords: VSC, HVDC, power systems, software-tosoftware validation, power electronics, electro-magnetic transients, DC grids, power systems

\section{Introduction}

\subsection{Motivation}

High Voltage Direct Current (HVDC) transmission systems have received renewed attention in the last decade due to their applications for long distance power transmission, particularly to enable interconnections between distant wind farms and the main electrical grid (Bahrman, 2006). There are two main converter technologies used in HVDCs: Line-Commutated Converter (LCC) and Voltage Source Converter (VSC), which are used for different applications in power systems (Abildgaard and Molinas, 2012). VSC-based HVDC systems provide certain advantages w.r.t. those based on LCC, including (Reed et al., 2003; Flourentzou et al., 2009), including independent control of active and reactive power,energy supply to weak and passive grids, etc.

An overview of different VSC topologies are reported in (Andersen et al., 2002) and include conventional twolevel, multi-level diode-clamped, floating capacitor multilevel converters, etc.

Recently, the Modular Multilevel Converter (MMC) technology has been adopted because of its advantages compared to other multilevel converter topologies for HVDC applications. With the adoption of MMC-based VSC technologies, modeling and simulation is becoming of crucial importance for different network studies; where modeling and simulation tools are needed in all facets related to their utilization: from design, through implementation, and in their operation.

\subsection{Related Works}

Power system electro-mechanical dynamic modeling and simulation is used for the analysis of dynamic behavior of large power networks, and the use of Modelica is now becoming attractive because of several advantages offered by the Modelica language as compared to existing power system simulation tools (Vanfretti et al., 2016; Casella et al., 2016). Another modeling and simulation approach that is important for power system analysis is the Electro-Magnetic Transient (EMT) methodology, and previous work has shown the advantages and limitations of the use of Modelica (Bachmann and Wiesmann, 2000) in adopting the EMT approach.

EMT analysis tools, such as EMTP-RV (see http:// emtp-software.com/), are typically used for the analysis of VSC-HVDC systems (Peralta et al., 2012), which allow to analyze their performance for different levels of modeling granularity of the power electronics of these systems (including average value models). To the knowledge of the authors, there only exists two previous implementations of VSC-HVDC models using the Modelica language in the literature (Majumder et al., 2013; Olenmark et al., 2015), however, these have not been implemented-in nor validated-against EMT (Electro-Magnetic Transient)-type power system simulation tools (e.g. EMTP-RV), and more importantly, they are not publicly available.

\subsection{Paper Contributions}

This paper reports the implementation of a three-phase VSC-HVDC average value model, and a power system test model that is compared against EMTP-RV. The aim is to show the potential use and challenges of applying the Modelica language for EMT-type analysis of VSCHVDC networks when detailed switching circuits do not need to be represented (e.g. system-level control design purposes). 
The remainder of this paper is organized as follows. Section 2 gives a brief description of the VSC-HVDC model. In Section 3, the model implementation in Modelica is explained, while in Section 4, software-to-software validation results are summarized. Finally, in Section 5, conclusions are drawn and future work is outlined.

\section{VSC-HVDC Model}

In EMTP-RV two types of VSC-based MMC station models are available, which are based on the results by (Peralta et al., 2012): Monopole, and Bipole configuration with ground return. The MMC stations are represented using four kinds of models: (a) Full detailed model, (b) Detailed equivalent model, (c) Switching function of arm model, and (d) Average-value model (AVM). The threephase configuration of the MMC topology assumed by these models is shown in Figure 1.

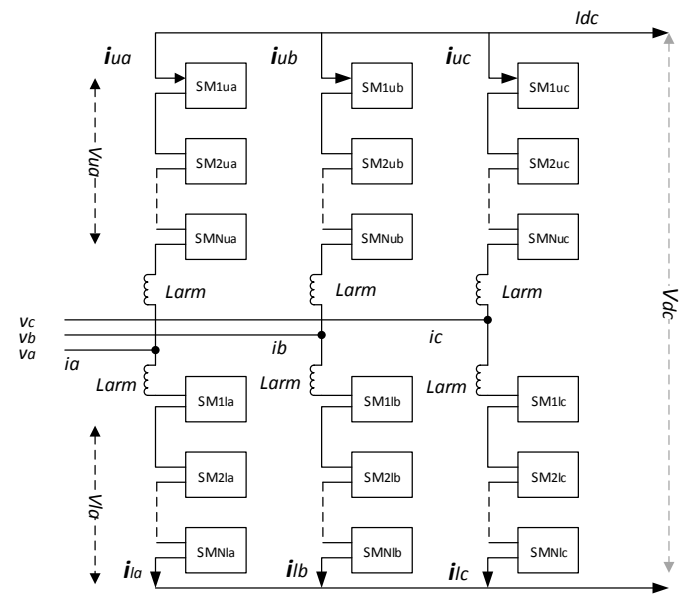

Figure 1. MMC topology.

In this work the AVM model with an high level control system is implemented. The full description of the model is documented in (Peralta et al., 2012). In this Section, the most relevant components of the model available in EMTP-RV are reviewed, as they are replicated in the Modelica implementation, in Section 3.

\subsection{Average-Value Model (AVM)}

In an AVM, the power electronic switches (IGBTs) and diodes are not modeled in detail, instead the MMC behavior is represented using controlled voltage and current sources. Thus, an ideal behavior of the internal variables of the MMC is assumed. For each phase $j=a, b, c$; the voltage of the converter is derived from Figure 1 , from where,

$$
v_{c o n v_{j}}=\frac{L_{a r m}}{2} \frac{d i_{j}}{d t}-v_{j}
$$

Assuming the total number of sub-modules in each phase is constant,

$$
v_{u_{j}}+v_{l_{j}}=V_{d c}
$$

where, $v_{u_{j}}$ and $v_{l_{j}}$ are upper and lower arm voltages. Using (1) and (2) the MMC is represented as a classical VSC. The controlled voltage source in the AC side is determined by:

$$
v_{\text {conv }_{j}}=v_{\text {ref }} \frac{V_{d c}}{2}
$$

where, $v_{r e f_{j}}$ are the reference voltages generated from the inner controller of the high level control system (i.e. they are dimensionless quantities in per unit). Based on the principle of power balance, the DC side model equations are derived assuming no energy is stored inside the MMC converter, as follows

$$
V_{d c} I_{d c}=\sum_{j=a, b, c} v_{\text {conv }_{j}} i_{j}
$$

where the DC side current is given by,

$$
I_{d c}=\frac{1}{2} \sum_{j=a, b, c} v_{r e f_{j}} i_{j}
$$

Using these principles, the AVM model implementation in EMTP-RV allows to build up an entire VSC-HVDC model. Figures 2 and 3 show the schematic of the implementation of the two basic components as available in EMTP-RV for this purpose.

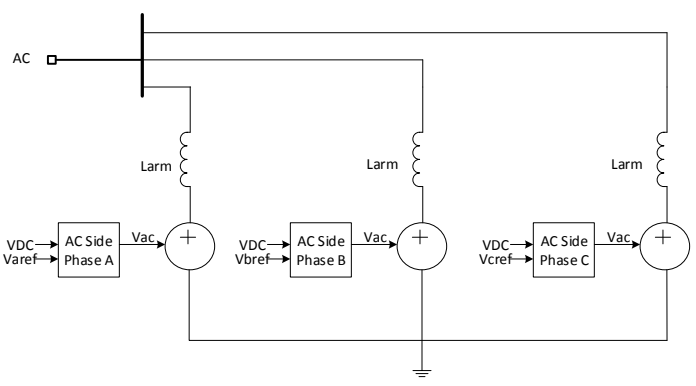

Figure 2. AC side of AVM model.

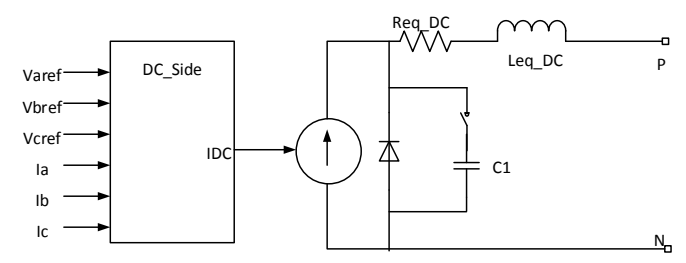

Figure 3. DC side of AVM Model.

In Figure 2 the AC side voltage for each phase is calculated using (3) and $L_{a r m}$ is the arm inductance. In Figure 3 the DC side current IDC is calculated using (5) and equivalent inductance, total conduction loss and the equivalent capacitor are given by, $L_{e q \_} D C=(2 / 3) L_{a r m}$, $R_{e q_{-} D C}=(2 / 3) N R_{O N}$ and $C 1=6 C / N$; where $N$ is the number of sub-modules per arm, $C$ is calculated using the energy conservation principle, and $R_{O N}$ represents the conduction loss of each IGBT. 


\subsection{Control System}

The VSC type MMC topology uses an "upper level" control system, which includes an outer and inner control. The structure of the overall "upper level" control system is shown in Figure 4. The "upper level" control system serves two main purposes: (i) to regulate "system variables", i.e. the active and/or reactive power or voltages (labeled "outer control" in Figure 4), and (ii) to generate reference voltages ( $v d \_r e f$ and $v q \_r e f$ ), which are used as input to the AVM.

\subsubsection{Upper Level Control}

The VSC-MMC model uses the classical vector control strategy. The inputs to the upper level control are threephase per unit (p.u.) variables, using the matrix in (8), these variables are converted to direct-and-quadrature-axis components rotating at synchronous speed $\left(\frac{d \theta}{d t}\right)$. The phase angle $\theta$ is calculated using a Phase-Locked Loop (PLL). The blocks for Clarke transformation, $\mathrm{P} / \mathrm{Q} / \mathrm{VAC}$ calculations and d-q transformation are used to compute the variables required for the outer and inner controllers. The $\mathrm{d}-\mathrm{q}$ transformed voltage and currents are calculated using the transformation matrix, $T$, as follows:

$$
\begin{array}{r}
i_{d q}=T i_{a b c} \\
v_{d q}=T v_{a b c \_g r i d}
\end{array}
$$

where

$$
T=\frac{2}{3}\left[\begin{array}{ccc}
\cos (\omega t) & \cos \left(\omega t-\frac{2 \pi}{3}\right) & \cos \left(\omega t+\frac{2 \pi}{3}\right) \\
-\sin (\omega t) & -\sin \left(\omega t-\frac{2 \pi}{3}\right) & -\sin \left(\omega t+\frac{2 \pi}{3}\right) \\
\frac{1}{2} & \frac{1}{2} & \frac{1}{2}
\end{array}\right] .
$$

The AC grid voltage, active and reactive power are calculated from the d-q reference,

$$
\begin{array}{r}
P=v_{d} i_{d}+v_{q} i_{q} \\
Q=-v_{d} i_{q}+v_{q} i_{d} \\
v_{\text {grid }}=\sqrt{v_{d}^{2}+v_{q}^{2}}
\end{array}
$$

The signals are converted to per unit (p.u.) quantities before entering to the upper level control system. The outer and inner control block is used to control active power, reactive power, DC and AC voltage. All these controllers are realized using proportional and integral (PI) control loop. The input to these PI controller loops are the difference between the reference (set by the user) and the controlled variable. The references to the outer control loop are usually fixed set points, that in practice are varied by a remote dispatcher. In this model the references to the outer control loop are fixed and can be varied by the user. The final block ( $d-q$ to $a b c)$ is used to convert the $d-q$ reference to three-phase voltage references.

\section{VSC Model Implementation in Modelica}

All the components included in the VSC model available in EMTP-RV are implemented in Modelica and described next. In addition to the VSC model, an equivalent generator model and a two winding transformer three-phase models were also implemented for software validation purposes.

\subsection{AVM Model in Modelica}

The AVM model was implemented using component models from the MSL (Modelica Standard Library). The connector models used in the AC side are the three-phase plug, and in DC side is the single phase positive and negative pin. The AVM model in Modelica is shown in Figure 5.

\subsection{Upper Level Control in Modelica}

Next, all the blocks of the upper level control system shown in Figure 4 were implemented in Modelica. As all the controllers in the upper level control system use the same PI controller implementation, first a PI controller using components from the MSL was implemented. Next the Modelica implementation was compared to the one implemented in EMTP-RV. After validating this component against EMTP-RV, the same PI controller was used in the remaining $\mathrm{P}, \mathrm{Q}, \mathrm{VDC}, \mathrm{VAC}$, inner control and PLL blocks.

\subsection{PLL in Modelica}

The phase locked loop (PLL) implemented in Modelica is shown in Figure 6. The main function of the PLL loop is to synchronize with the phase angle and frequency of the AC grid voltage. The implementation of the PLL used similar components in Modelica, as those available in the specific power system tool's documentation/description (i.e. EMTP-RV). Given the fact that the reference documentation has a copyright, so the detail description is not given here.

\section{Software-to-Software Validation}

\subsection{Sub-system Model Validation}

The AVM and each block of the upper level control system were implemented as individual models within one package, then all the blocks were assembled to realize complete the implementation of the VSC model.

Next, software-to-software validation was carried out against the EMTP-RV model. For example, consider the PLL block shown in Figure 6, it has two inputs and two outputs. After the implementation of the entire PLL block in Modelica, this model was validated by simulating it using the same input signals in both Modelica and EMTP$\mathrm{RV}$. The results of the PLL block simulations are shown in Figures 7 and 8 .

The same procedure is followed for the three-phase equivalent generator and three-phase two winding transformer models shown in Figure 9. After implementing needed components, a test power system model described next is used to validate the VSC-HVDC model. 


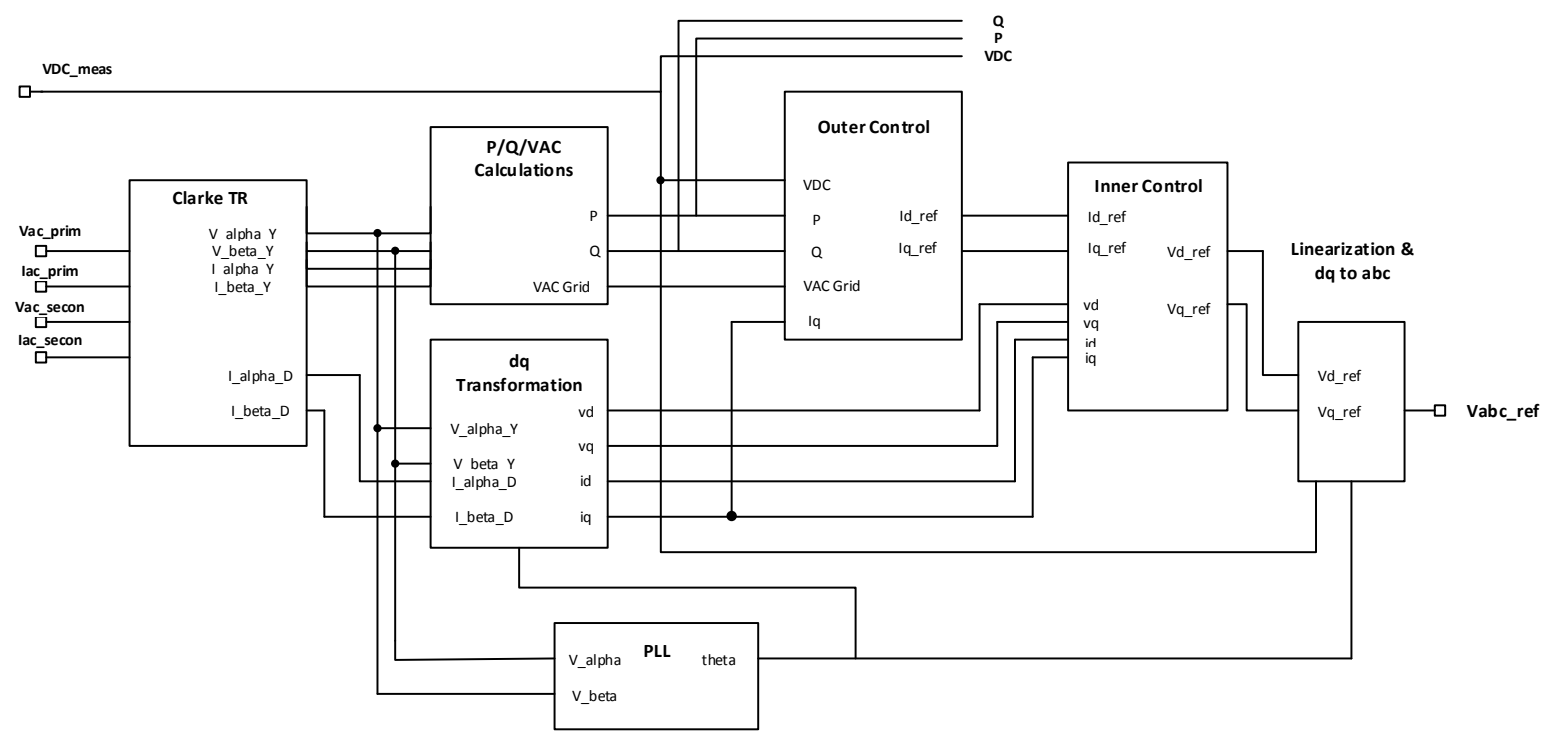

Figure 4. Block diagram of the control system of the VSC-HVDC.

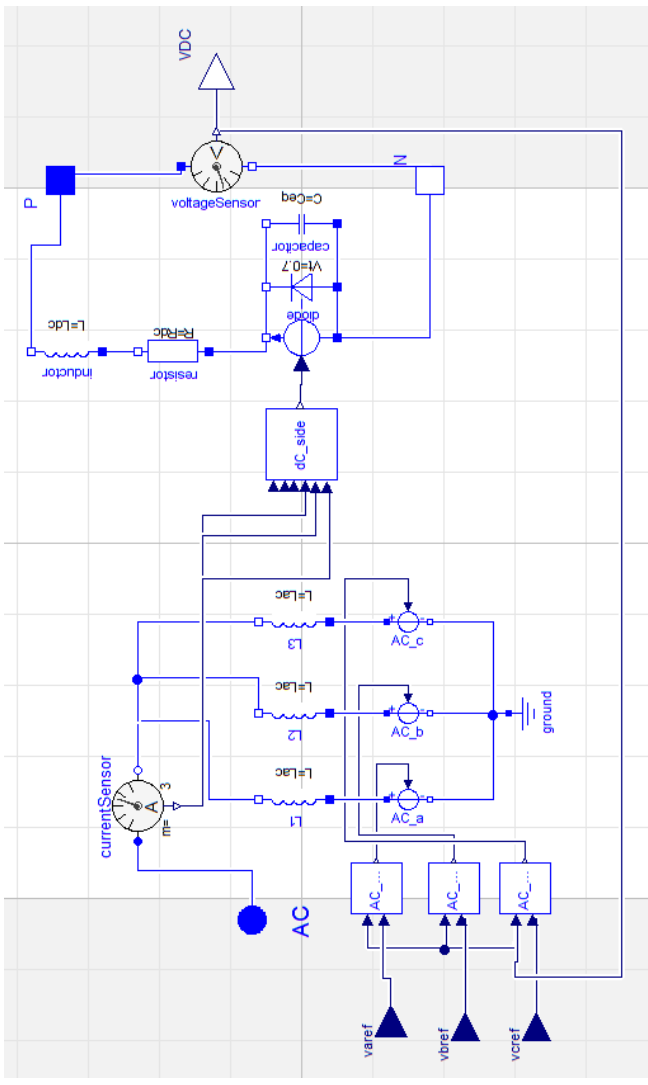

Figure 5. AVM Model in Modelica.

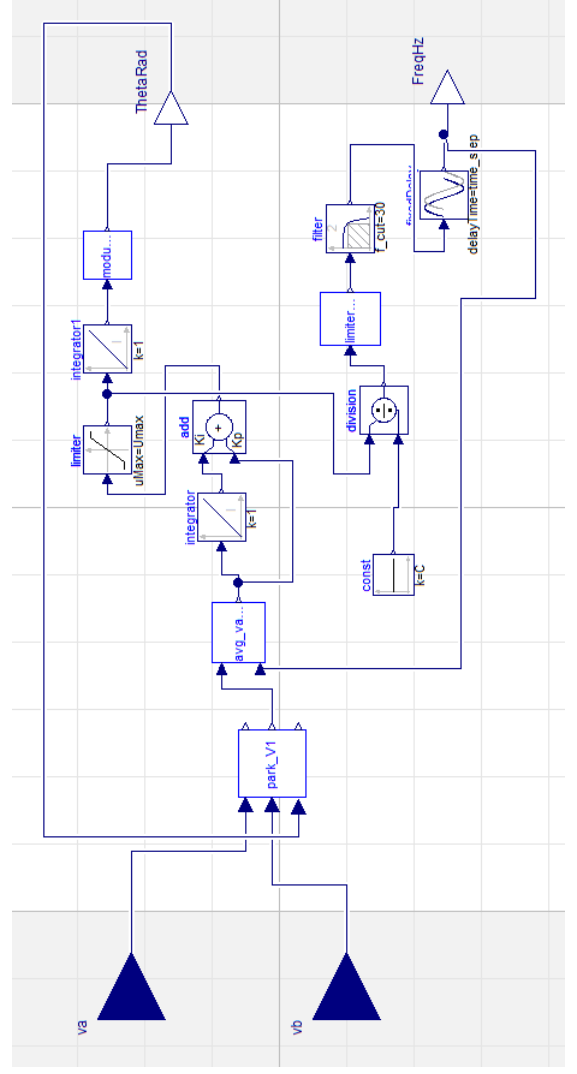

Figure 6. PLL in Modelica.

\subsection{Power System Test model}

The VSC-HVDC test power system model implemented in Modelica and EMTP-RV is shown in Figure 10. A DC cable model is yet to be implemented in Modelica, and thus, resistive line model $(R=1.022 \Omega)$ is used instead. Converter 1 (VSC1) controls the active power and Con- verter 2 (VSC2) controls the DC voltage, while $1000 \mathrm{MW}$ active power is transferred from VSC1 to VSC2. The user can select which controller should be active in each VSC. The model parameters used (i.e. transformer resistance and reactance, MMC arm inductance, etc), are exactly the same in both software tools, and are summarized in 

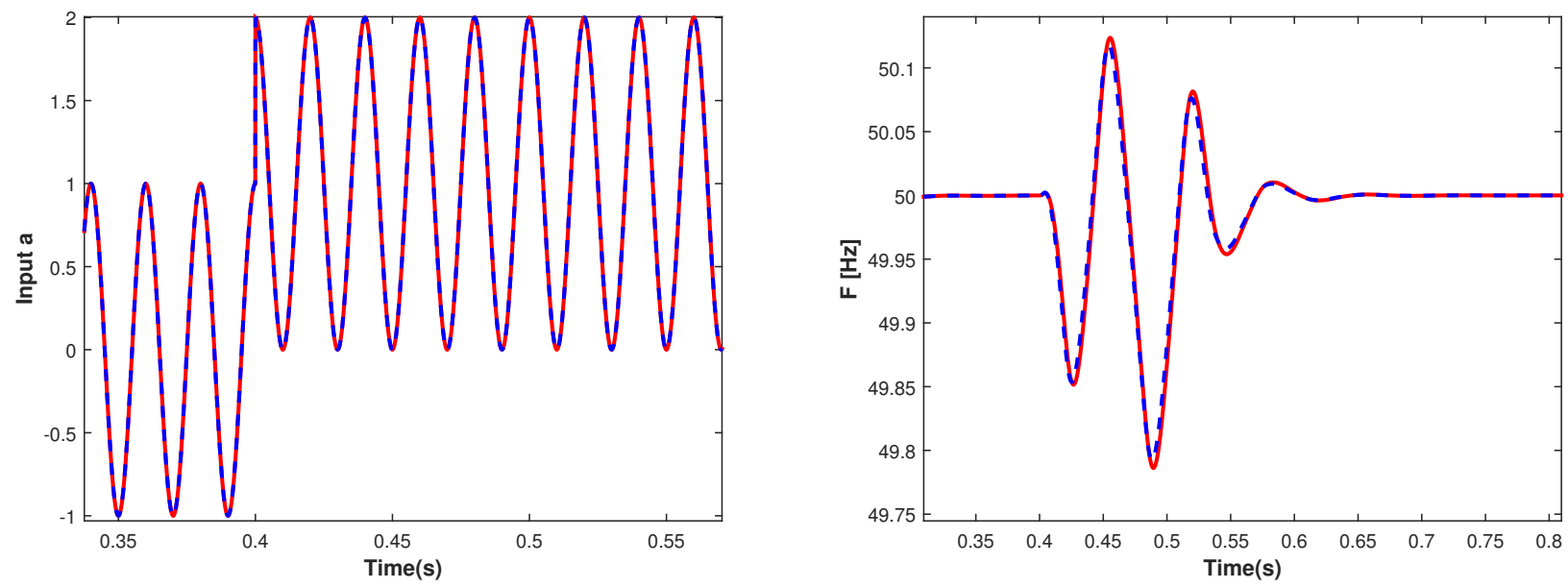

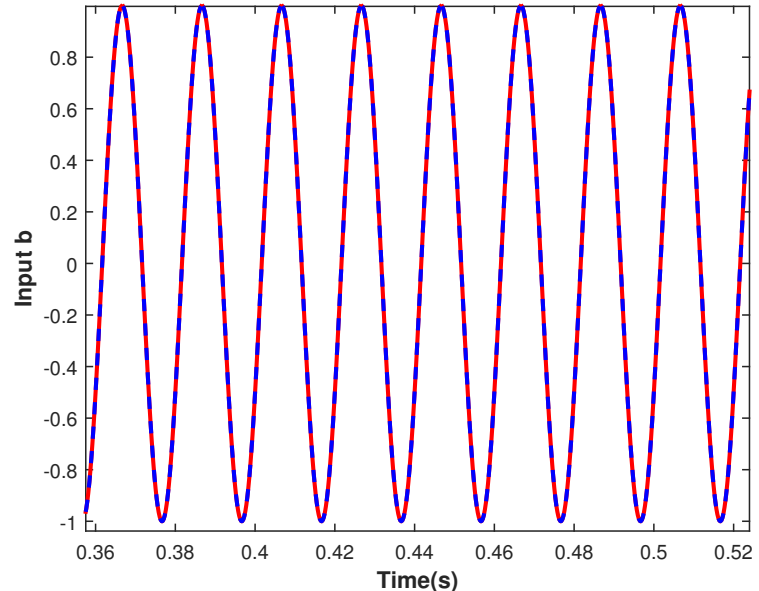

Figure 7. Inputs to the PLL block. (Red traces: EMTP-RV and blue traces: Modelica)

the Appendix. In EMTP-RV the integral and proportional gains of each PI controller (in upper level control system) are automatically calculated by specifying the desired settling time (with 5\% error). The computation method used by EMTP-RV is proprietary, and thus, for the sake of consistency, the values computed by this tool are used in the Modelica model.

\subsection{Steady State}

EMTP-RV initializes the model variables using a threephase power flow solver, which is not available outside of this tool. To validate the sub-system models (i.e. equivalent generator, controllers, PLL, etc.), no initialization values were provided to the Modelica models (starting values of the voltage and currents were set $=0$ ). At the beginning of the simulation, the Modelica and EMTP-RV results do not match, however, after the Modelica trajectories reach in their steady-state, the simulation results show an adequate match. To illustrate, consider the test system shown in Figure 9, where an equivalent generator and three-phase two winding transformer are included. No initialization values for the inductor currents (i.e. the states) were pro-

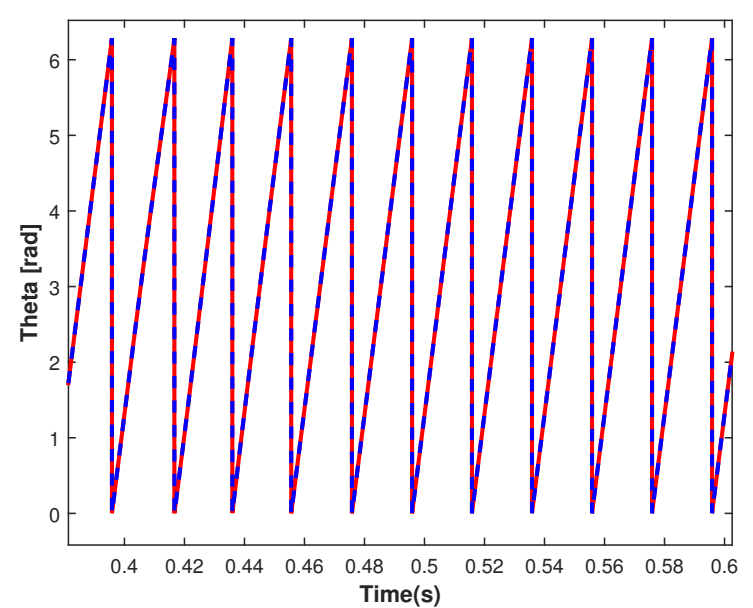

Figure 8. Outputs of the PLL block. (Red traces: EMTP-RV and blue traces: Modelica)

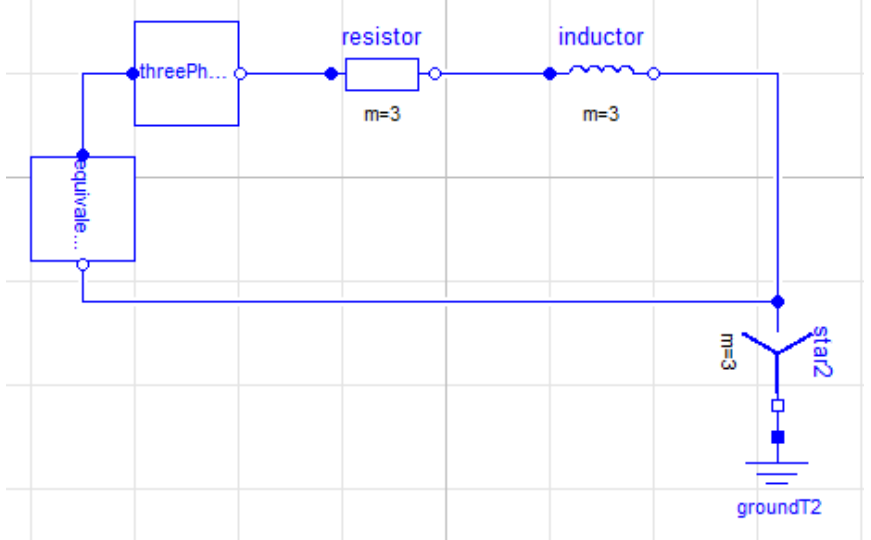

Figure 9. Test system of the equivalent generator and the two winding transformer.

vided.

The simulation was carried out using the solver Dass 1 with interval length equal to $1 e-5$. The same interval length is used in EMTP-RV, however note that EMTP-RV uses a Trapezoidal solver.

The simulation results shown in Figure 11 show that at 


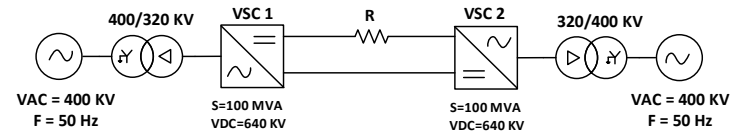

Figure 10. VSC-HVDC Test system.

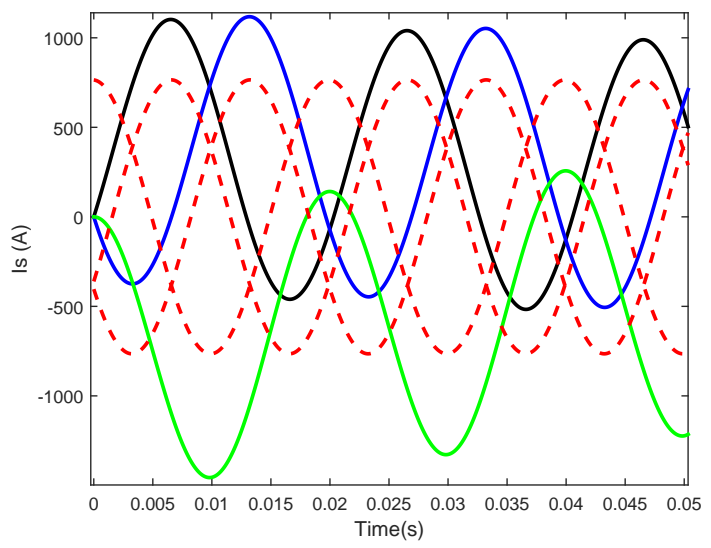

Figure 11. Secondary current of the transformer before the steady-state is reached (Red: EMTP-RV, other: Modelica).

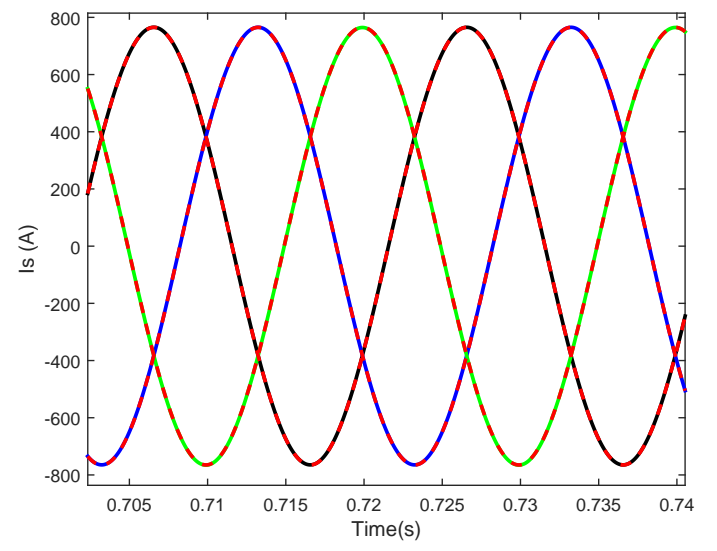

Figure 12. Secondary current of the transformer when the steady-state is reached.

the beginning of the simulation the traces do not match for the two different implementations. The traces in red are from EMTP-RV, while other traces in different colors are from the Modelica tool used. The simulation output of the Modelica model matches the EMTP-RV results after the steady-state is reached (shown in Figure 12).

Observe that when a larger test system model is to be simulated (see Section 4.2), there are more states that need to be initialized. The authors found that some of the Modelica-tools (OpenModelica and Dymola), the solvers are not able to solve the initialization problem and/or to execute the simulation successfully. For the test system shown in Figure 10 the Rkfix 4 solver with interval length $1 e-5$ and tolerance of 0.01 were used.

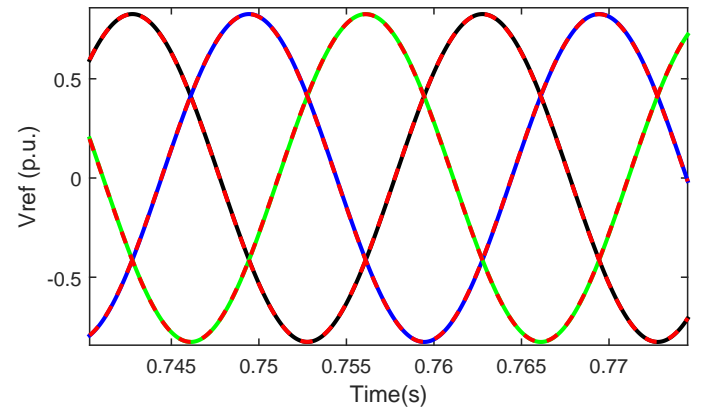

Figure 13. $V_{a b c \text { ref }}$ of VSC1 i.e. output of upper level control (Red: EMTP-RV, other: Modelica).

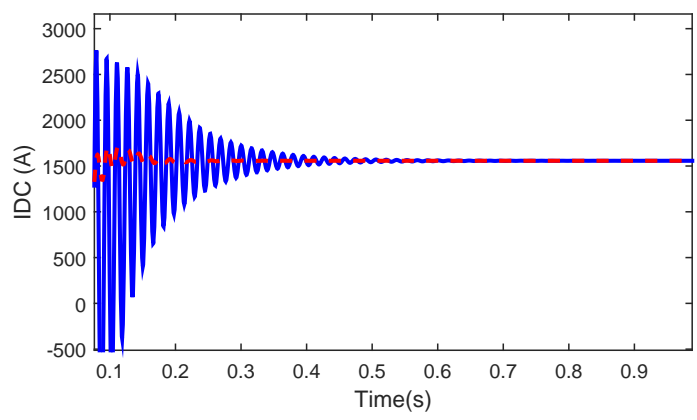

Figure 14. DC current of VSC1 (Red: EMTP-RV, Blue: Modelica).

\subsection{Software-to-Software Validation}

Software-to-Software validation of the VSC-HVDC model (see Figure 10) is carried out in two steps. First, simulations are carried out without applying any perturbations to the model in order to check whether the steadystate trajectories match or not. In addition, no initial values were provided to the controller's integrators in the Modelica model. As a result, the simulation is allowed to reach the steady state value before disturbances are applied and comparisons are made. Figures 13 and 14 show the simulation results for $V_{a b c \_r e f}$ and IDC of VSC1, showing the close match between the two different implementations.

Next, a step change in the active power reference form 1 to 0.5 (1000 MW to $500 \mathrm{MW}$ ) at 0.8 second is applied. The step change and response of the controller are shown in Figure 15, while other trajectories are shown in Figures 16-19. It is noted that a close match is achieved between both implementations.

\section{Conclusion}

This paper showed the potential use of the Modelica language to model EMT-type models of VSC-HVDC systems when the high-frequency switching process can be represented using equivalent voltage and current sources. Differently from the authors previous work (Vanfretti et al., 2017), this model is built using as much components as possible from the MSL to represent the three-phase elec- 


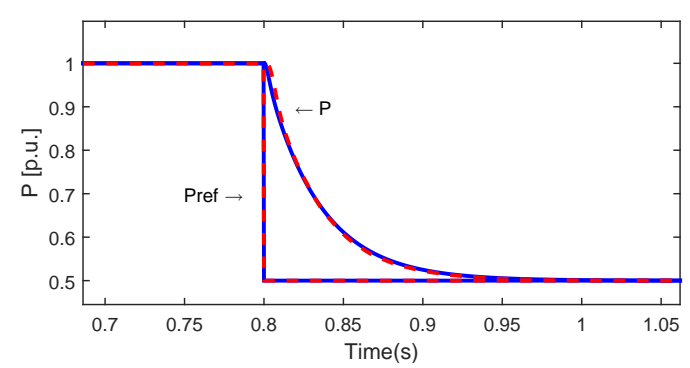

Figure 15. Active power response of VSC1 (Red: EMTP-RV, Blue: Modelica).

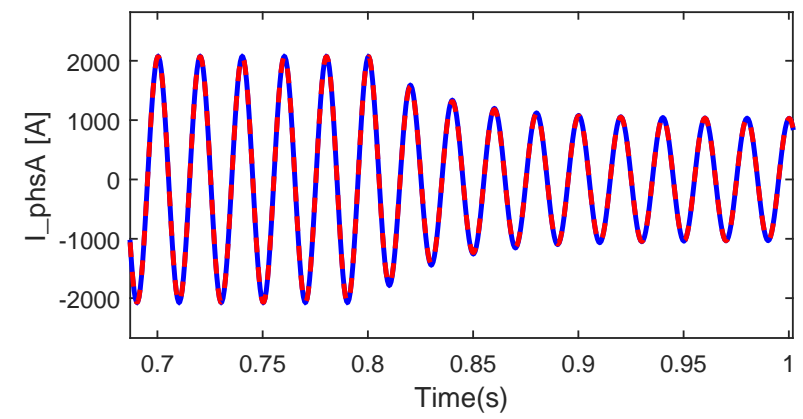

Figure 16. Primary current (Phase A) of VSC1 (Red: EMTPRV, Blue: Modelica).

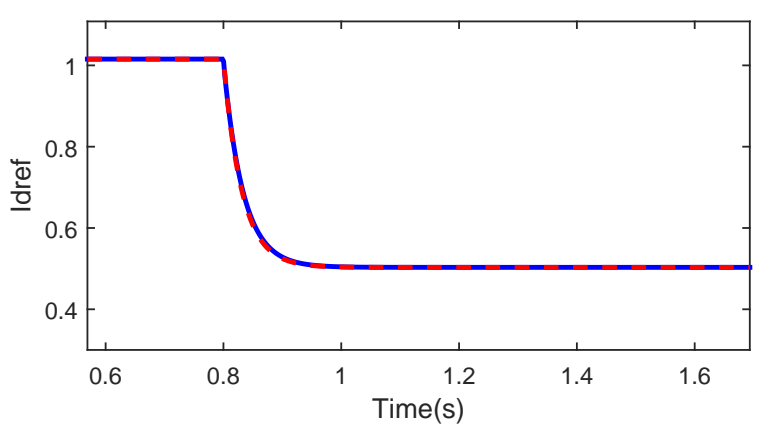

Figure 17. Current reference of upper level control of VSC1 (Red: EMTP-RV, Blue: Modelica).

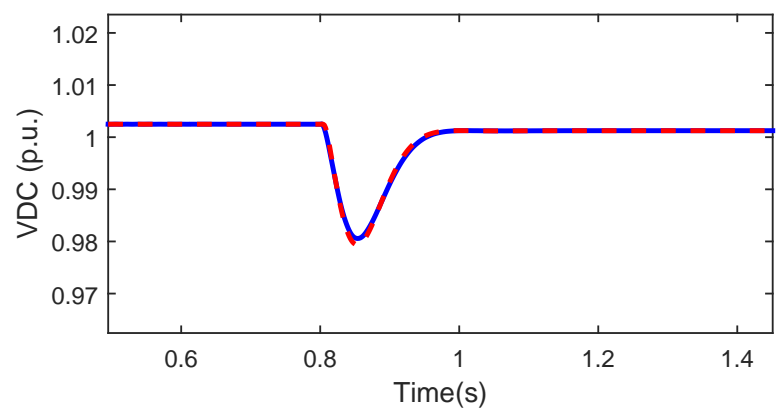

Figure 18. DC voltage on the VSC1 side (Red: EMTP-RV, Blue: Modelica).

trical circuit, while implementing the de facto control system models used within typical power system simulation tools.

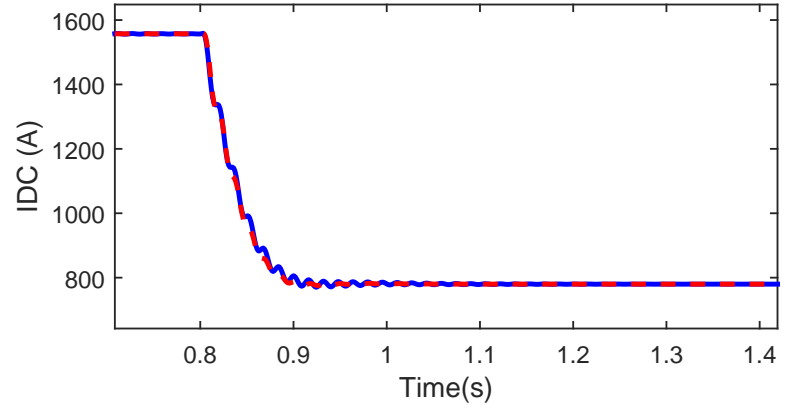

Figure 19. DC current on the VSC1 side (Red: EMTP-RV, Blue: Modelica).

The Modelica implementation was compared to the EMTP-RV software, a de facto power system modeling and simulation tool used for VSC-HVDC analyses, yielding surprisingly similar results (even identical when a desired disturbance is applied after the steady-state is reached). The major benefit of the work reported herein is that the control system implemented can now be exchanged with different tools that support the FMI standard, including Simulink and EMTP-RV, which makes it possible to keep and maintain a single version of the control system model implemented (i.e. the one in Modelica).

The results from this work show that there is great potential for the use of Modelica for EMT-type modeling and simulation of electrical power systems, and particularly of power electronic components. However, further work must be carried out with respect to the provision of adequate starting guess values for the initialization problem, and more importantly, to efficiently simulate switching processes without substantially affecting simulation time.

The Modelica files of the model presented in this paper are available under the GPLv3 license in the following GitHub repository: https://github.com/SmarTS-Lab/2017_ ModelicaConf_VSC-HVDC_AVM_Model

\section{Acknowledgment}

This work was supported in part by the FP7 iTesla project, the ITEA3 openCPS project, and the STandUP Collaboration Initiative.

Mohammed Ahsan Adib Murad is supported by Science Foundation Ireland under Grant No. SFI/15/IA/3074.

The authors would like to thank Professor Federico Milano for supporting the first author during the preparation of this paper.

\section{Appendix}

The two node test power system model parameter data are provided in the Tables 1 and 2.

\section{References}

E. N. Abildgaard and M. Molinas. Modelling and control of the Modular Multilevel Converter (MMC). En- 
Table 1. Equivalent generator data.

\begin{tabular}{ll}
\hline Parameter & Value \\
\hline Line to line RMS voltage (KV) & 400 \\
Generator short circuit capacity (MVA) & 10000 \\
R/L ratio (p.u.) & 10 \\
Fequency (Hz) & 50 \\
\hline
\end{tabular}

Table 2. VSC and two winding transformer data.

\begin{tabular}{ll}
\hline Parameter & Value \\
\hline Rated power (MVA) & 1000 \\
$\begin{array}{l}\text { Transformer primary voltage [r.m.s. LL] } \\
\text { (KV) }\end{array}$ & 400 \\
Transformer secondary voltage [r.m.s. & 320 \\
LL] (KV) & \\
Fequency (Hz) & 50 \\
Transformer resistance (p.u.) & 0.001 \\
Transformer reactance (p.u.) & 0.18 \\
MMC arm inductance (p.u.) & 0.15 \\
Capacitor energy in each sub-module & 40 \\
(KJ/MVA) & 400 \\
Number of sub-module per arm, N & .001 \\
Conduction loss of each IGBT (p.u.) & \\
\hline
\end{tabular}

ergy Procedia, 20:227 - 236, 2012. ISSN 1876-6102. doi:http://dx.doi.org/10.1016/j.egypro.2012.03.023.

B. R. Andersen, L. Xu, P. J. Horton, and P. Cartwright. Topologies for VSC transmission. Power Engineering Journal, 16(3):142-150, June 2002. ISSN 0950-3366. doi:10.1049/pe:20020307.

B. Bachmann and H. Wiesmann. Advanced modeling of electromagnetic transients in power systems. In Modelica Workshop 2000 , Oct 2000.

M. P. Bahrman. Overview of HVDC transmission. In 2006 IEEE PES Power Systems Conference and Exposition, pages 18-23, Oct 2006. doi:10.1109/PSCE.2006.296221.

F. Casella, A. Bartolini, S. Pasquini, and L. Bonuglia. Objectoriented modelling and simulation of large-scale electrical power systems using Modelica: A first feasibility study. In IECON 2016 - 42nd Annual Conference of the IEEE Industrial Electronics Society, pages 6298-6304, Oct 2016. doi:10.1109/IECON.2016.7793558.

N. Flourentzou, V. G. Agelidis, and G. D. Demetriades. VSC based HVDC power transmission systems: An overview. IEEE Transactions on Power Electronics, 24(3):592-602, March 2009. ISSN 0885-8993. doi:10.1109/TPEL.2008.2008441.

R. Majumder, B. Berggren, and M. Larsson. Development and comparison of DC grid model in Powerfactory and Dymola for controller design. In 2013 IEEE Power Energy Society General Meeting, pages 1-5, July 2013. doi:10.1109/PESMG.2013.6672328.
A. Olenmark, J. Sloth, A. Johnsson, C. Wilhelmsson, and J. Svensson. Control development and modeling for flexible DC grids in Modelica. In 2015 The 11th International Modelica Conference, September 2015.

J. Peralta, H. Saad, S. Dennetiere, J. Mahseredjian, and S. Nguefeu. Detailed and averaged models for a 401level MMC-HVDC system. IEEE Transactions on Power Delivery, 27(3):1501-1508, July 2012. ISSN 0885-8977. doi:10.1109/TPWRD.2012.2188911.

G. Reed, R. Pape, and M. Takeda. Advantages of voltage sourced converter (VSC) based design concepts for FACTS and HVDC-link applications. In 2003 IEEE Power Engineering Society General Meeting (IEEE Cat. No.03CH37491), volume 3, page 1821 Vol. 3, July 2003. doi:10.1109/PES.2003.1267437.

L. Vanfretti, T. Rabuzin, M. Baudette, and M. Murad. itesla power systems library (iPSL): A Modelica library for phasor time-domain simulations. SoftwareX, 5:84 - 88, 2016. ISSN 2352-7110. doi:http://dx.doi.org/10.1016/j.softx.2016.05.001.

L. Vanfretti, M.A.A. Murad, and F.J.Gómez. Calibrating a VSC-HVDC model for dynamic simulations using RaPId and EMTP simulation data. In 2017 IEEE Power Energy Society General Meeting, pages 1-5, July 2017. 